\title{
Analysis of indications for pulmonary peripheral lesions diagnosed using radial endobronchial ultrasound-guided transbronchial lung biopsy
}

\author{
SHU-HONG GUAN, MING ZHANG, SU-JUAN ZHANG, QIU-DI ZHANG, QIAN-QIAN XU and JUN ZHOU
}

Department of Respiratory and Critical Care Medicine, Changzhou No. 1 Hospital, Changzhou, Jiangsu 213000, P.R. China

Received September 29, 2018; Accepted January 10, 2020

DOI: $10.3892 /$ etm.2020.8734

\begin{abstract}
The aim of the present study was to determine the indications for radial endobronchial ultrasound-guided transbronchial lung biopsy (rEBUS-D-TBLB) for the diagnosis of peripheral pulmonary lesions (PPL) located at the bronchopulmonary segments and subsegments. Data collected from 774 patients who underwent rEBUS-D-TBLB for suspected PPL, including clinical information, distribution of lesions, diagnostic spectrum and diagnostic rate, were collected and retrospectively reviewed. Additionally, the Wilcoxon signed-rank test was performed to analyze the diagnostic yield of lesions in bronchopulmonary subsegments under the lesion diameter limit of $3 \mathrm{~cm}$. In total, 802 lesions were found in 774 patients. The diagnostic yield of rEBUS-D-TBLB for all lesions was $67.18 \%$. Overall, 362 cases of malignant disease and 158 cases of benign disease were diagnosed, with sensitivities of 70.98 and $79.00 \%$ respectively. Lesions were distributed throughout the 18 bronchopulmonary segments of the lungs. The bronchopulmonary segments with $>5 \%$ of the majority of the discovered lesions were LB1+2, LB3, LB6, LB10, RB1-4 and RB9. The diagnostic yield of rEBUS-D-TBLB was found to be $>65 \%$ for lesions located at LB3, RB1-3 and RB9. Further rEBUS-D-TBLB examinations of the LB1+2a, LB6a and RB4b segments produced diagnostic yields of 81.25, 66.67 and $71.43 \%$ respectively. Finally, at segment RB4a, rEBUS-D-TBLB examination was more effective for lesions with diameters $>3 \mathrm{~cm}$ compared with lesions with diameters $<3 \mathrm{~cm}$. The diagnostic yields for PPL distributed at LB1+2a, LB3, LB6a, RB1-3, RB4a (diameter $>3 \mathrm{~cm}$ ), RB4b, and RB9 using rEBUS-D-TBLB were higher compared with for other segments, providing a theoretical basis for the clinical application of rEBUS-D-TBLB for the diagnosis of PPL in patients.
\end{abstract}

Correspondence to: Dr Jun Zhou, Department of Respiratory and Critical Care Medicine, Changzhou No. 1 Hospital, 185 Ju-qian Street, Changzhou, Jiangsu 213000, P.R. China

E-mail: yunxu1969@163.com

Key words: pulmonary peripheral lesions, radial endobronchial ultrasound-guided transbronchial lung biopsy, indications, diagnosis, bronchopulmonary segments

\section{Introduction}

Lung cancer is becoming one of the most prevalent malignancies in China, which is associated with a particularly high morbidity (17.1\% of cancers) and high mortality $(21.6 \%$ of cancer-related deaths) (1). Smoking, environmental pollution, and fumes from fuel burning are among the reported risk factors for this disease $(1,2)$. The majority of lung cancer cases are diagnosed at the latter stages, which precludes curative surgery and leads to poor prognosis (3). In China, the estimated five-year survival rate for lung cancer is only $16.1 \%$ (4). To promote the early diagnosis and subsequent therapeutic intervention of lung cancer, low-dose spiral computed tomography (CT) is used as a screen. However, peripheral pulmonary lesions (PPL), which are pre-cursors to lung cancer, occasionally develop in positions that are difficult to visualize, which increases the difficulty of CT-guided diagnosis.

Recently, minimally invasive methods, including percutaneous transthoracic and thoracoscopic biopsy, have been commonly applied for the diagnosis of PPL $(5,6)$. However, limitations persist with such techniques, including the risk of pneumothorax and bleeding. Radial endobronchial ultrasound-guided transbronchial lung biopsy (rEBUS-D-TBLB) potentially offers a major contribution to the diagnosis of PPL (7).

Theoretically, although the entire chest cavity is within the detection range of ultrasound, the diagnostic positive rate by rEBUS-TBLB is not constant throughout the chest in clinical practice $(8,9)$. To increase the diagnostic yield of rEBUS-TBLB for PPL, analysis of its indications at different locations of the lung would be of particular clinical significance. Therefore, the present study retrospectively analyzed the data collected from 774 patients with PPL, to explore the indications in using rEBUS-D-TBLB for the diagnosis of PPL at various bronchopulmonary segments and subsegments, providing a theoretical basis for its clinical application.

\section{Materials and methods}

Subjects. The present retrospective study was approved by the Ethics Committee of Changzhou No. 1 Hospital (Changzhou, China). All patients provided written informed consent. 
Data were collected from 774 patients (sex, 516 males and 258 females; age range, 26-85 years; mean age, $62.81 \pm 9.19$ years) who underwent rEBUS-D-TBLB at the Department of Respiratory Medicine, Changzhou No. 1 Hospital between January 1, 2015 and August 31, 2017. General clinical data of the patients are shown in Table I.

The inclusion criterion was as follows: Patients with lesions located below the opening of segmental bronchi as revealed by chest $\mathrm{CT}$. The exclusion criteria were as follows: i) Patients without a blurred opaque shadow of bronchial structure or pulmonary vascular sign on chest high resolution CT; ii) patients with lesions that were revealed by routine bronchoscopy; and iii) patients with contraindications for bronchoscopy, including hemorrhagic tendency, active respiratory hemorrhage, severe cardiopulmonary insufficiency, and incapacity to cooperate. A schematic of the selection criteria for the present study is shown in Fig. 1.

Materials. The following equipment was used: BF-P260 flexible bronchoscope; EU-ME1 ultrasonic mainframe; MAJ-935 ultrasonic probe driving unit; UM-S20-17S $20 \mathrm{MHz}$ ultrasonic miniprobe $(1.4 \mathrm{~mm})$; and UM-S20-20R $20 \mathrm{MHz}$ ultrasonic miniprobe $(1.7 \mathrm{~mm})$. All equipment was purchased from Olympus Corporation.

$r E B U S-D-T B L B$. Prior to the rEBUS-D-TBLB operation, results of the $C T$ scan, including the diameter and localization of the lesion, were carefully recorded; there was also a record of the bronchial path through which the ultrasonic probe was to reach the lesion.

After $6 \mathrm{~h}$ preoperative fasting and water deprivation, the patients were anesthetized through inhalation of lidocaine for $5 \mathrm{~min}$. A routine bronchoscopy was conducted to search for and assess the lesion; rEBUS-D-TBLB was subsequently performed in cases where no definite lesion was found. Using the imaging system as a guide, the ultrasonic probe was delivered to the inspection location through the biopsy channel. On meeting resistance, the ultrasonic scan was initiated, and the ultrasonic probe was slowly pulled backwards; changes in the ultrasonic images were closely observed until a clear ultrasonic image of the bronchial wall where the lesion is located becomes visible. The adjacent bronchi were then inspected to determine the optimal site for performing the biopsy. After the ultrasonic probe was withdrawn, a pair of biopsy forceps was inserted which followed the same path down to the site of the lesion, guided by the ultrasonic distance measurements $(10,11)$. No more than eight biopsies were performed until ideal diagnostic rates, accurate subtypes and molecular detection were obtained. If no lesion was detected by the ultrasound probe within $30 \mathrm{~min}$, rEBUS was terminated and was considered ineffective for that case. All patients suspected to have PPL were all diagnosed with PPL.

Final diagnosis. Pathological diagnoses of chronic mucosal inflammation, fibrous hyperplasia and idioblast (cells different in shape, size, structure and contents of surrounding cells) were all considered negative for PPL, whilst every diagnosis of suspected cancer was considered positive for PPL.

If the use of data from rEBUS-D-TBLB could not reach a diagnosis, other methods were performed, including
Table I. Clinical data from the patients.

Indicators

No. of cases $[\mathrm{n}(\%)]$

Sex

Male

516

Female

258

Age

$\leq 60$ years 288

$>60$ years 486

TNM stage

IA

$25(2.23)$

IIB

$29(3.75)$

IIA

$16(2.01)$

IIB

$11(1.42)$

IIIA

$63(8.14)$

IIIB

IVA

$183(23.64)$

TNM, tumor-node-metastasis.

transthoracic needle biopsy, surgical operation, metastatic biopsy or follow-up, to find the definitive diagnosis.

Statistical analysis. Statistical analysis was performed using SPSS 16.0 software (SPSS, Inc). Measurement data are presented as the mean $\pm \mathrm{SD}$ and enumeration data as number/percentage (n/\%). The Wilcoxon signed-rank sum test was used to analyze the diagnostic yield of lesions in the subsegments that are below the lesion diameter limit of $3 \mathrm{~cm} . \mathrm{P}<0.05$ was considered indicate a statistically significant difference.

\section{Results}

Diagnoses of the 774 patients with PPL using rEBUS-D-TBLB. In the $774 \mathrm{PPL}$ patients, there were 802 lesions, 336 in the left lung and 466 in the right. The diagnostic yield of rEBUS-D-TBLB for all patients was $67.18 \%$ (520/774). Overall, 362 cases of malignant disease, including adenocarcinoma and squamous carcinoma, were found, whilst 158 cases of benign diseases were diagnosed; the diagnostic sensitivity of malignancy was $70.98 \%$ (181/255 patients) and the diagnostic sensitivity of benign tumors was $79.00 \%$ (79/100 patients).

Pathological findings in the 520 patients with PPL diagnosed by $r E B U S-D-T B L B$. Through rEBUS-D-TBLB, the histopathological findings of 520 PPL patients were obtained from the biopsies, including 362 cases of malignant disease and 158 cases of benign disease.

Among the 362 cases of malignant diseases adenocarcinoma was the most common at 260 cases, accounting for $71.28 \%$ of all the malignant diseases. There were 66 cases of squamous carcinoma (18.23\%), 10 cases with malignant tumors not pathologically classified $(2.76 \%), 8$ cases of adenosquamous carcinoma (2.21\%), 4 cases of small-cell carcinoma and 4 cases of mucosa-associated lymphoid tissue lymphoma. 


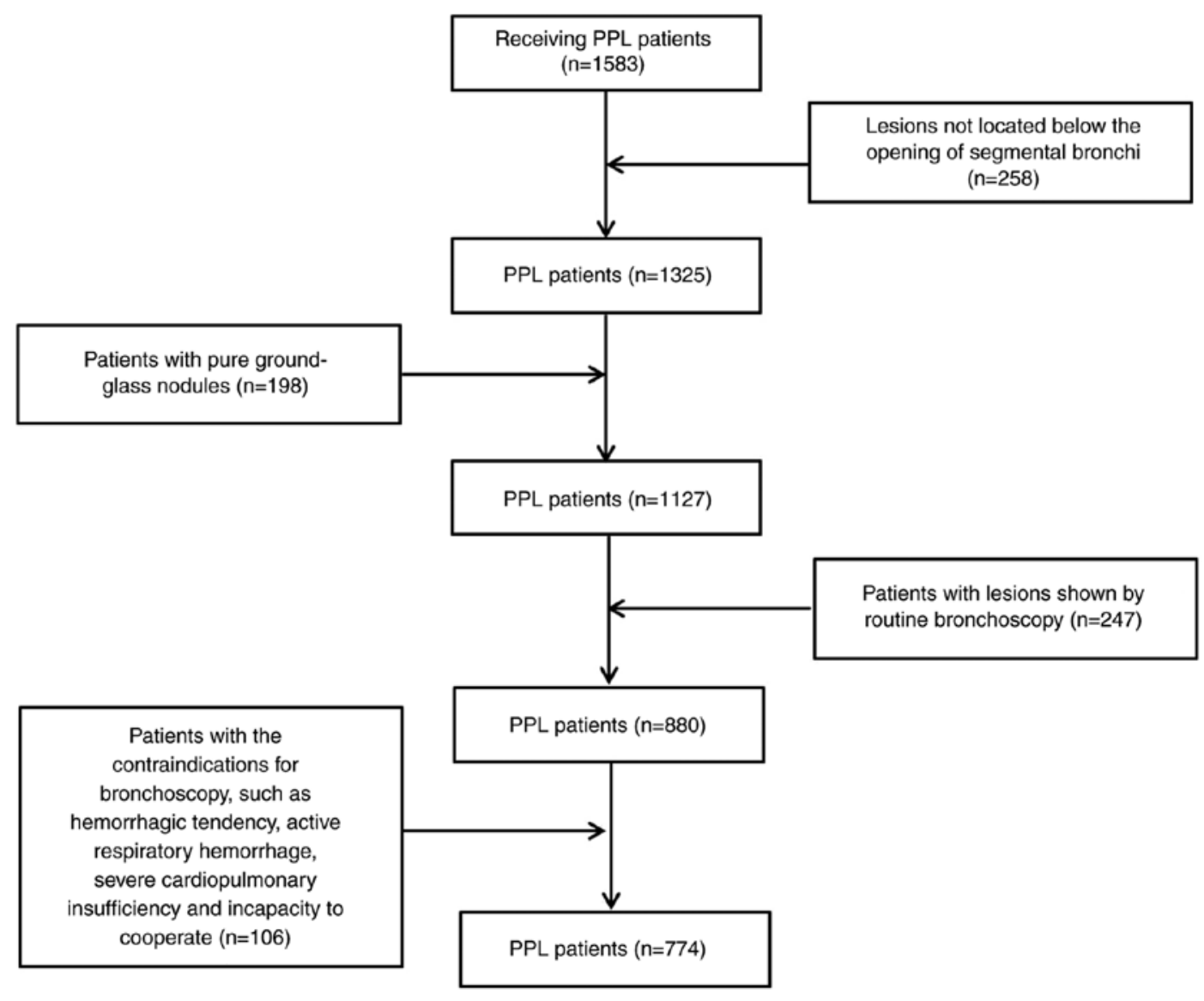

Figure 1. Schematic of patient selection for the present study. PPL, peripheral pulmonary lesions.

Additionally, 2 cases each of spindle cell malignancy, sarcomatoid carcinoma, adenocarcinoma combined with sarcomatoid carcinoma, adenocarcinoma combined with small cell carcinoma and poorly-differentiated neuroendocrine carcinoma were found (Table II).

Among the 158 cases of benign disease, nonspecific pneumonia ( 72 cases, $45.57 \%$ ) was the most frequently diagnosed, followed by tuberculosis (32 cases, 20.25\%). There were 18 cases of inflammatory myofibroblastic tumor (11.39\%), 16 cases of lung abscess (10.13\%), 6 cases each of pulmonary aspergillosis and pulmonary cryptococcosis $(3.80 \%), 4$ cases of organizing pneumonia $(2.53 \%)$ and 2 cases each of pulmonary mucormycosis and radiation pneumonitis (1.27\%; Table III).

Distribution of lesions among the bronchopulmonary segments. The 802 lesions were distributed in all 18 bronchopulmonary segments of the bilateral lungs (Fig. 2). Sorting by the number of lesions found in each bronchopulmonary segment with $>5 \%$ of lesions gave the following results: 106 lesions were found in the posterior segment of the right upper lobe bronchus (RB2; 13.22\%), 98 in the apical posterior segment of the left upper lobe bronchus (LB1+2;12.22\%), 88 in the apical segment of the right upper lobe bronchus (RB1; 10.97\%), 68 in the anterior segment of the left upper lobe bronchus (LB3; 8.48\%), 54 in the anterior segment of the right upper lobe bronchus (RB3; 6.73\%), 46 in the lateral segment of the middle lobe bronchus (RB4; 5.74\%), 42 in the superior segment of the left lower lobe bronchus (LB6; 5.24\%),
42 in the posterior basal segment of the left lower lobe bronchus (LB10; 5.24\%) and 42 in the lateral basal segment of right lower lobe bronchus (RB9; 5.24\%).

Detectivity and diagnostic ability of $r E B U S-D-T B L B$ in the 802 lesions. 'Detected' refers to the ability of rEBUS to detect the lesion and obtain the tissue through rEBUS-D-TBLB, with negative pathological diagnosis results. 'Diagnosed' refers to the ability of rEBUS to detect the lesion and obtain the tissue through rEBUS-D-TBLB, with positive pathological diagnosis results. According to the aforementioned results, 106 of the lesions were found in the RB2 segment, in which rEBUS-D-TBLB detected 98 (92.45\%) of these and diagnosed $80(75.47 \%)$. In the RB3 segment, where 54 lesions were found, rEBUS-D-TBLB detected $50(92.95 \%)$ and diagnosed 38 (70.37\%). There were 88 lesions in the RB1 segment, where rEBUS-D-TBLB detected $74(84.09 \%)$ and diagnosed $60(68.18 \%)$. In the LB3 segment, 68 lesions were confirmed, where rEBUS-D-TBLB detected $62(91.18 \%)$ and diagnosed $46(67.75 \%)$. In the RB9 segment, 42 lesions were found, where rEBUS-D-TBLB detected 40 (95.24\%) and diagnosed 28 (66.67\%). The lesion detection rates for other bronchopulmonary segments were all found to be $>80 \%$, whereas the rates of diagnosis were considered low at $<65 \%$ (Fig. 3).

Lesion analysis in the bronchopulmonary LB1+2, LB6, LB10 and RB4 subsegments. Although $>5 \%$ of the 802 lesions were found in the LB1+2, LB6, LB10 and RB4 segments, the 
Table II. Pathological findings in 362 cases of malignant disease.

\begin{tabular}{lc}
\hline Diagnosis & No. of cases $(\%)$ \\
\hline Adenocarcinoma & $260(71.28)$ \\
Squamous carcinoma & $66(18.23)$ \\
Malignant tumors not pathologically & $10(2.76)$ \\
classified & \\
Adenosquamous carcinoma & $8(2.21)$ \\
Small cell carcinoma & $4(1.10)$ \\
Mucosa associated lymphoid tissue & $4(1.10)$ \\
lymphoma & \\
Spindle cell malignancy & $2(0.55)$ \\
Sarcomatoid carcinoma & $2(0.55)$ \\
Adenocarcinoma combined with & $2(0.55)$ \\
sarcomatoid carcinoma & \\
Adenocarcinoma combined with small & $2(0.55)$ \\
cell carcinoma & \\
Poorly differentiated neuroendocrine & $2(0.55)$ \\
carcinoma &
\end{tabular}

Table III. Pathological findings in 158 cases of benign diseases

\begin{tabular}{lc}
\hline Diagnosis & No. of cases $(\%)$ \\
\hline Pneumonia & $72(45.57)$ \\
Tuberculosis & $32(20.25)$ \\
Inflammatory pseudotumor & $18(11.39)$ \\
Lung abscess & $16(10.13)$ \\
Pulmonary aspergillosis & $6(3.80)$ \\
Pulmonary cryptococcosis & $6(3.80)$ \\
Organizing pneumonia & $4(2.53)$ \\
Pulmonary mucormycosis & $2(1.27)$ \\
Radiation pneumonitis & $2(1.27)$ \\
\hline
\end{tabular}

capability of rEBUS-D-TBLB to diagnose the lesions was lower in these segments compared with other segments. Therefore, the lesions found in these bronchopulmonary subsegments were analyzed further to confirm the diagnostic yields.

The diagnostic yields of the lesions were comparatively high; the yield in the LB1+2 apical branch $(\mathrm{LB} 1+2 \mathrm{a})$ was found to be $81.25 \%$, whereas that in the LB6 superior branch (LB6a) was 66.67 and $71.43 \%$ in the RB4 internal branch (RB4b). The diagnostic yields in the LB6 external branch (LB6b), LB10 posterior branch (LB10a), LB10 external branch (LB10b) and LB10 internal branch (LB10c) were found to be low, with few lesions. The diagnostic yields in the $\mathrm{LB} 1+2$ posterior branch (LB1+2b), LB1+2 external branch $(\mathrm{LB} 1+2 \mathrm{c}), \mathrm{LB} 6$ internal branch (LB6c) and RB4 external branch (RB4a) were also found to be low, although many lesions were found (Fig. 4).

Analysis of diagnostic yield using Wilcoxon signed-rank test. Wilcoxon signed-rank test was used to analyze the diagnostic yields of rEBUS-D-TBLB between lesions with a diameter of $\geq 3$ and $<3 \mathrm{~cm}$ at the LB1+2b, LB1+2c, LB6c and RB4a segments. The overall diagnostic yields of rEBUS-D-TBLB for lesions were lower compared with other segments. The diagnostic yields could not be increased by distinguishing the size of lesions in $\mathrm{LB} 1+2 \mathrm{~b}, \mathrm{LB} 1+2 \mathrm{c}$ or LB6c segments with the exception of RB4a, in which the diagnostic yield of rEBUS-D-TBLB was higher for lesions with a diameter of $>3 \mathrm{~cm}$ compared with that for $<3 \mathrm{~cm}$ (Table IV).

\section{Discussion}

A number of techniques have been developed for diagnosing PPL over recent decades, including blind bronchoscopic examination, and thoracic, thoracoscopic and percutaneous transthoracic biopsies. However, these techniques are associated with limitations. Blind bronchoscopic examination has a low success rate (12), whereas thoracic and thoracoscopic biopsies result in significant trauma in patients $(13,14)$. For percutaneous transthoracic biopsy, although it remains the principal approach for the PPL diagnosis, it is less effective for lesions near the major vessels of the heart and diaphragm, in addition to those distant from the body surface $(15,16)$; importantly, percutaneous transthoracic biopsy is performed through the non-natural lumina, leading to a high incidence of postoperative complications such as pneumothorax and bleeding (17). Hospitalization is therefore required for all patients undergoing percutaneous transthoracic biopsy, increasing the treatment cost and the use of medical resources (18).

With the development of ultrafine bronchoscopes and endobronchial ultrasound, rEBUS-TBLB is becoming increasingly important for diagnosing PPL. is conducted through the natural lumina of the tracheal bronchus, a safe route that causes minimal damage (19). Additionally, the positive diagnostic rate increases when it is combined with other technologies, including X-ray fluoroscopy, guiding sheath and virtual navigation. A previous meta-analysis demonstrated that the diagnostic yield of rEBUS-TBLB was as high as $69 \%$, with only $27 \%$ of the postoperative complications experienced with percutaneous transthoracic biopsy (20). The use of rEBUS-TBLB is extended due to its high diagnostic yield and superior safety. The ultrasonic characteristics of rEBUS-TBLB lesions have high clinical value, as parameters including lesion properties, airway structure and filtration range can be characterized. A previous report revealed that rEBUS-TBLB and transbronchial brushing confer advantages in the evaluation of PPL, but cannot access lesions located adjacent to the proximal segmental bronchus (21). The high point specificity (95\% CI=0.99-1.00) and point sensitivity (95\% CI=0.70-0.76) of rEBUS-TBLB renders it suitable for the diagnosis of peripheral lung cancer (22). Additionally, the application of transbronchial needle aspiration has drastically improved the diagnostic yield, especially when the EBUS probe cannot reach the PPL (23). A previous study has shown that the diagnostic yield of EBUS-guided bronchoscopy alone was $69.0 \%, 50.6 \%$ when combined with TBLB, $42.0 \%$ when combined with brush smear and $44.3 \%$ in combination with bronchoalveolar lavage fluid analysis (24). The present study found the diagnostic yield of rEBUS-D-TBLB for all lesions 


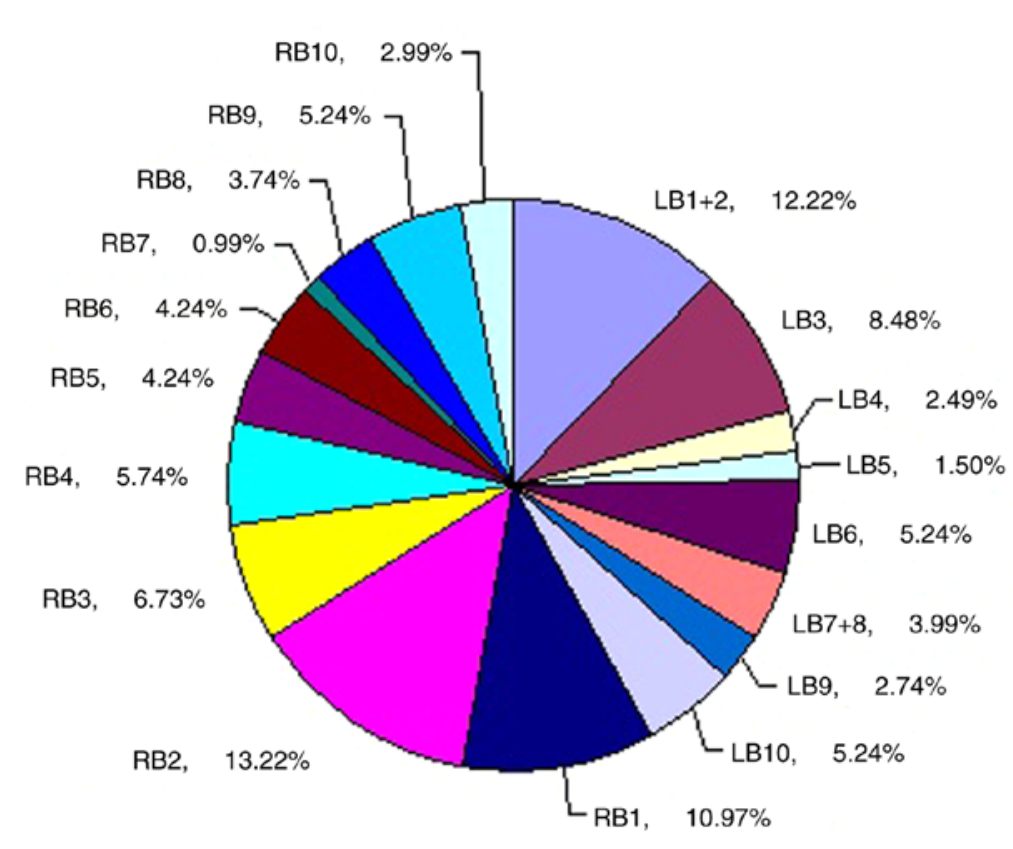

\begin{tabular}{|l|}
\hline$\square$ LB1+2 \\
$\square$ LB3 \\
$\square$ LB4 \\
$\square$ LB5 \\
$\square$ LB6 \\
$\square$ LB7+8 \\
$\square$ LB9 \\
$\square$ LB10 \\
$\square$ RB1 \\
$\square$ RB2 \\
$\square$ RB3 \\
$\square$ RB4 \\
$\square$ RB5 \\
$\square$ RB6 \\
$\square$ RB7 \\
$\square$ RB8 \\
$\square$ RB9 \\
$\square$ RB10 \\
\hline
\end{tabular}

Figure 2. Distribution of the 802 lesions found in the respective bronchopulmonary segments using radial endobronchial ultrasound. RB2, posterior segment of right upper lobe bronchus; LB1+2, apical posterior segment of left upper lobe bronchus; RB1, apical segment of right upper lobe bronchus; LB3, anterior segment of left upper lobe bronchus; RB3, anterior segment of right upper lobe bronchus; RB4, lateral segment of middle lobe bronchus; LB6, superior segment of left lower lobe bronchus; LB10, posterior basal segment of left lower lobe bronchus; RB9, lateral basal segment of right lower lobe bronchus.

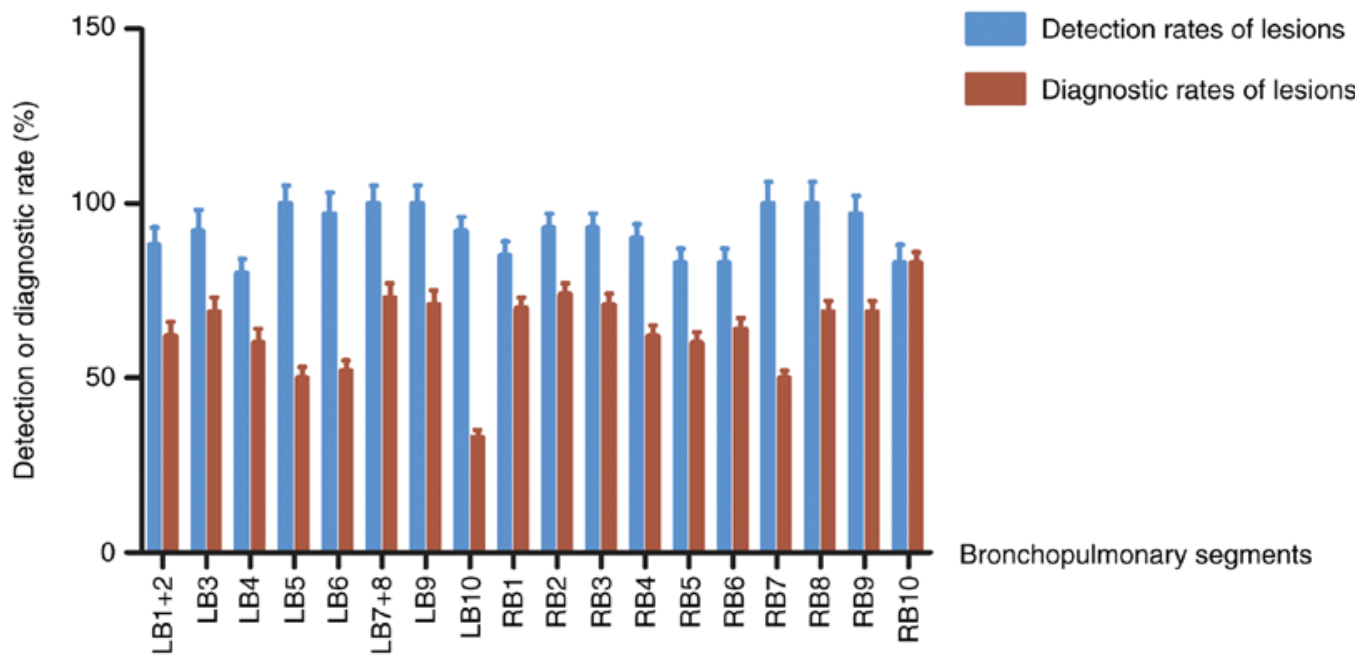

Figure 3. Rates of lesion detection by radial endobronchial ultrasound and diagnostic ability of subsequent transbronchial lung biopsy in the 401 lesions found in the corresponding bronchopulmonary segments. RB2, posterior segment of right upper lobe bronchus; LB1+2, apical posterior segment of left upper lobe bronchus; RB1, apical segment of right upper lobe bronchus; LB3, anterior segment of left upper lobe bronchus; RB3, anterior segment of right upper lobe bronchus; RB4, lateral segment of middle lobe bronchus; LB6, superior segment of left lower lobe bronchus; LB10, posterior basal segment of left lower lobe bronchus; RB9, lateral basal segment of right lower lobe bronchus.

to be $67.18 \%$, consistent with this previous observation (24). In addition, the present study also found the diagnostic yield of rEBUS-D-TBLB in the LB1+2a segment to be $81.25 \%$, in comparison with $66.67 \%$ in the LB6a segment and $71.43 \%$ in RB4b the segment, suggesting that rEBUS-D-TBLB confers advantages for the diagnosis of lesions in LB1+2a compared with percutaneous lung puncture.
The outer diameter $(1.4-2.0 \mathrm{~mm})$ of the rEBUS probe allows it to reach into the sixth subsegmental bronchus with a scan range of $4 \mathrm{~cm}$ (19). In ideal situations, all lesions in the pulmonary field should be detectable. However, the diagnostic ability of rEBUS-TBLB was lower compared with percutaneous transthoracic biopsy (25). In clinical practice, the positive diagnostic rate of rEBUS-TBLB was significantly 
Table IV. Analysis of diagnostic rates of lesions using Wilcoxon signed-rank test.

\begin{tabular}{|c|c|c|c|c|c|c|c|}
\hline Subsegments & $\begin{array}{c}\text { Diagnostic } \\
\text { rates of lesions (\%) }\end{array}$ & Subgroups & $\begin{array}{l}\text { Number } \\
\text { of lesions }\end{array}$ & $\begin{array}{l}\text { Diagnostic } \\
\text { rates }\end{array}$ & $\mathrm{t}$ & $\mathrm{t}$ range & P-value \\
\hline \multirow[t]{2}{*}{$\mathrm{LB} 1+2 \mathrm{~b}$} & \multirow[t]{2}{*}{57.89} & $<3 \mathrm{~cm}$ & 16 & $50 \%(8 / 16)$ & \multirow[t]{2}{*}{64.5} & \multirow[t]{2}{*}{$55-105$} & \multirow[t]{2}{*}{$\mathrm{P}>0.05$} \\
\hline & & $\geq 3 \mathrm{~cm}$ & 22 & $63.64 \%(14 / 22)$ & & & \\
\hline \multirow{2}{*}{$\mathrm{LB} 1+2 \mathrm{c}$} & \multirow[t]{2}{*}{42.86} & $<3 \mathrm{~cm}$ & 14 & $28.57 \%(4 / 14)$ & \multirow[t]{2}{*}{46.5} & \multirow[t]{2}{*}{$36-69$} & \multirow[t]{2}{*}{$\mathrm{P}>0.05$} \\
\hline & & $\geq 3 \mathrm{~cm}$ & 14 & $57.14 \%(8 / 14)$ & & & \\
\hline \multirow[t]{2}{*}{ LB6c } & \multirow[t]{2}{*}{50} & $<3 \mathrm{~cm}$ & 6 & $0 \%(0 / 6)$ & \multirow[t]{2}{*}{17} & \multirow[t]{2}{*}{$7-26$} & \multirow[t]{2}{*}{$\mathrm{P}>0.05$} \\
\hline & & $\geq 3 \mathrm{~cm}$ & 14 & $100 \%(14 / 14)$ & & & \\
\hline \multirow[t]{2}{*}{ RB4a } & \multirow{2}{*}{56.25} & $<3 \mathrm{~cm}$ & 18 & $22.22 \%(4 / 18)$ & \multirow[t]{2}{*}{38.5} & \multirow[t]{2}{*}{$40-79$} & \multirow[t]{2}{*}{$\mathrm{P}<0.05$} \\
\hline & & $\geq 3 \mathrm{~cm}$ & 14 & $100 \%(14 / 14)$ & & & \\
\hline
\end{tabular}

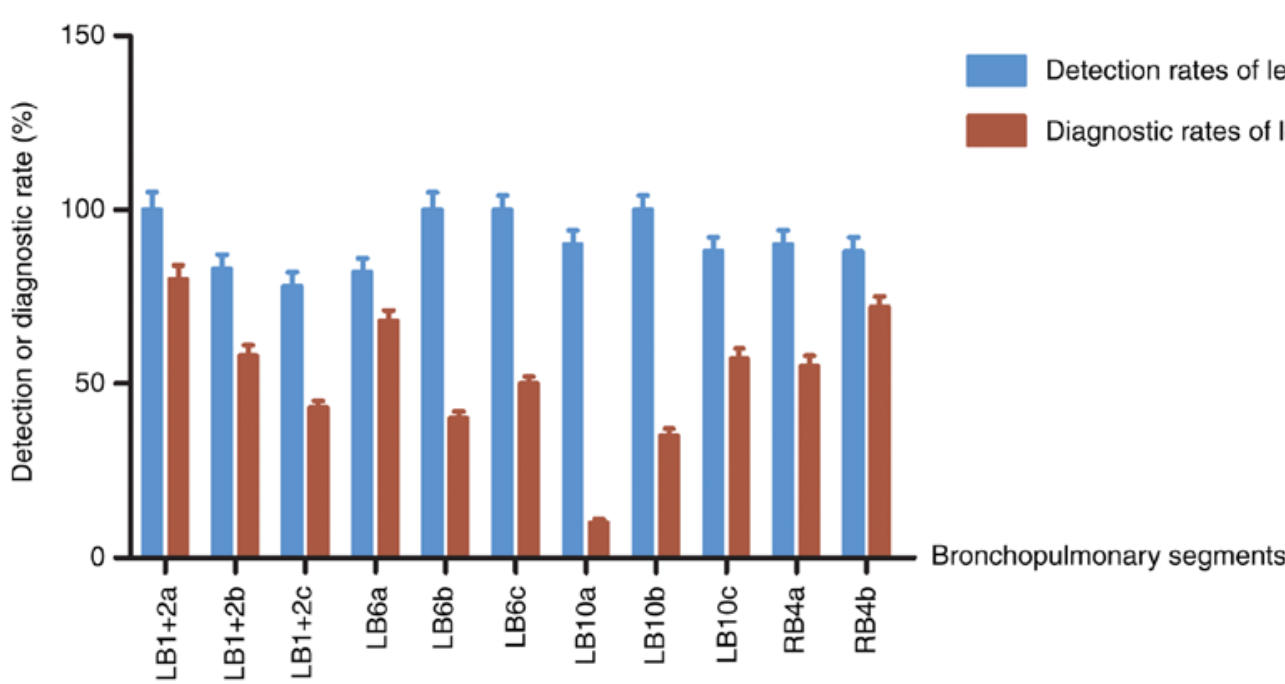

Figure 4. Rates of lesion detection by radial endobronchial ultrasound and diagnostic ability of subsequent transbronchial lung biopsy in lesions located at the bronchopulmonary LB1+2, LB6, LB10 and RB4 subsegments. LB1+2a, LB1+2 apical branch; LB1+2b, LB1+2 posterior branch; LB1+2c, LB1+2 external branch; LB6a, LB6 superior branch; LB6b, LB6 external branch; LB6c, LB6 internal branch; LB10a, LB10 posterior branch; LB10b, LB10 external branch; LB10c, LB10 internal branch; RB4a, RB4 external branch; RB4b, RB4 internal branch.

lower in bronchiolar segments compared with others. Although rEBUS-TBLB detected central lesions with typical ultrasonic images, the positive diagnostic rate remained low, possibly due to the degree of curvature of the rEBUS-TBLB path to the lesion and variable sensitivities of lesions with differing properties to pathological examination. To increase the diagnostic rate of PPL by rEBUS-TBLB, analysis of indications at each bronchopulmonary segment and subsegment would be of marked clinical benefit, since information regarding this issue is lacking. Previous studies have demonstrated the effects of lesion location on the positive diagnostic rate of rEBUS-TBLB at the pulmonary lobe level. One study reported that distinguishing lesions did not improve the positive diagnostic rate of rEBUS-TBLB (10), whilst another study showed that the diagnostic rates for lesions in the left upper pulmonary lobe were demonstratively lower compared with those for lesions in other lobes (9). Another previous finding demonstrated that although the diagnostic rates of lesions in the right middle lobe and left lingular lobe were relatively high, the diagnostic rates of lesions between the right middle lobe and left lingular lobe had no significant difference (26). In the present study, therefore, the sample size was increased to 774 patients, allowing for further exploration of the indications for rEBUS-D-TBLB in the diagnosis of PPL at different locations in the lung.

In the present study, 802 lesions were found in 774 patients. The diagnostic yield of rEBUS-D-TBLB for all patients was $67.18 \%$, which lies within the range of that reported by previous studies $(27,28)$. In addition, through rEBUS-D-TBLB, 362 cases of malignant diseases and 158 cases of benign diseases were identified with sensitivities of 70.98 and $79.00 \%$, respectively. This high percentage demonstrates the importance of rEBUS-D-TBLB for pathological diagnosis, which can potentially prevent false positive diagnoses, facilitating prompt treatment and improved prognoses.

To study the indications for rEBUS-D-TBLB in the diagnosis of PPL at the bronchopulmonary segments and subsegments, the general distribution of lesions in the whole pulmonary field was first examined. The results showed that the 802 lesions were distributed throughout the 18 bronchopulmonary segments of the bilateral lungs. Those with $>5 \%$ of lesions were LB1+2, LB3, LB6, LB10, RB1, RB2, RB3, RB4 and RB9, whereas other bronchopulmonary segments found 
with fewer lesions were not explored further in the present study.

As previously reported, the diagnostic yield of rEBUS-TBLB for PPL was found to be $>65 \%$ (29). According to this criterion, rEBUS-TBLB is recommended for bronchopulmonary segments with $>5 \%$ of lesions and rEBUS-TBLB diagnostic yields for PPL of $>65 \%$. In the aforementioned nine bronchopulmonary segments, the diagnostic yields in RB2, RB3, RB1, LB3 and RB9 by rEBUS-TBLB were $>65 \%$. However, those in the four remaining bronchopulmonary segments with $>5 \%$ of lesions, namely LB1+2, LB6, LB10 and RB4, were found to be low. Therefore, lesions in these four bronchopulmonary subsegments were analyzed further to improve the diagnostic yield. It was found that the rEBUS-TBLB diagnosis yields for PPL in subsegments LB1+2a (81.25\%), LB6a (66.67\%) and RB4b $(71.43 \%)$ were sufficient for the recommendation of rEBUS-TBLB. The rEBUS-TBLB diagnostic yields for PPL in LB6b, LB10a, LB10b and LB10c subsegments were low with small numbers of lesions, which were not studied further. In the LB1+2b, LB1+2c, LB6c and RB4a subsegments, although the diagnostic rates were low, a number of lesions were found. Therefore, the Wilcoxon signed-rank test was subsequently performed to analyze the rEBUS-TBLB diagnostic yields further for PPL under the lesion diameter limit of $3 \mathrm{~cm}$ in the LB1+2b, LB1+2c, LB6c and RB4a segments. The diagnostic yields in LB1+2b, LB1+2c and LB6c cannot be increased by distinguishing the size of lesion, though the diagnostic ability of rEBUS-D-TBLB was comparatively high for lesions $>3 \mathrm{~cm}$ in diameter in $\mathrm{RB} 4 \mathrm{a}$ compared with those $<3 \mathrm{~cm}$.

A number of limitations remain attached to the present study. Although it presents the diagnostic rates of lesions by rEBUS-D-TBLB, which provide a theoretical basis for the diagnosis of PPL using rEBUS-D-TBLB, the present study did not associate the pathological findings with the clinical endpoints. In addition, the Wilcoxon signed-rank test was performed to analyze the diagnostic yield of lesions, but not multivariate or univariate analysis, which may be more suitable for statistical comparisons across different parameters.

In conclusion, the diagnostic yields of rEBUS-D-TBLB for PPL, especially those distributed at the $\mathrm{LB1}+2 \mathrm{a}, \mathrm{LB} 3$, LB6a, RB1, RB2, RB3, RB4a (diameter $>3 \mathrm{~cm}$ ), RB4b and RB9 subsegments, were higher compared with those for other segments, providing the theoretical basis for the clinical application of rEBUS-D-TBLB in the diagnosis of PPL.

\section{Acknowledgements}

Not applicable.

\section{Funding}

The present study was supported by the guiding science and technology project of the Health Planning Commission of Changzhou in 2017 (grant no. WZ201710).

\section{Availability of data and materials}

All data generated or analyzed during this study are included in this published article.

\section{Authors' contributions}

GSH and ZJ designed this study. SHG, JZ and ZM performed the experiments and collected the data. GSH, ZSJ, ZQD and XQQ analyzed the data. GSH wrote the manuscript. All authors read, revised and approved the manuscript.

\section{Ethics approval and consent to participate}

The present retrospective study was approved by the Ethics Committee of Changzhou No. 1 Hospital (Changzhou, China).

\section{Patient consent for publication}

Not applicable.

\section{Competing interests}

The authors declare that they have no competing interests.

\section{References}

1. Chen W, Zheng R, Baade PD, Zhang S, Zeng H, Bray F, Jemal A, Yu XQ and He J: Cancer statistics in China, 2015. CA Cancer J Clin 66: 115-132, 2016.

2. Zheng R, Zeng H, Zhang S and Chen W: Estimates of cancer incidence and mortality in China, 2013. Chin J Cancer 36: 66, 2017.

3. Wong MCS, Lao XQ, Ho KF, Goggins WB and Tse SLA: Incidence and mortality of lung cancer: Global trends and association with socioeconomic status. Sci Rep 7: 14300, 2017.

4. Zeng H, Zheng R, Guo Y, Zhang S, Zou X, Wang N, Zhang L, Tang J, Chen J, Wei K, et al: Cancer survival in China, 2003-2005: A population-based study. Int J Cancer 136: 1921-1930, 2015.

5. Wang T, Luo L and Zhou Q: Risk of pleural recurrence in early stage lung cancer patients after percutaneous transthoracic needle biopsy: A meta-analysis. Sci Rep 7: 42762, 2017.

6. Luo K, Lin Y, Lin X, Yu X, Wen J, Xi K, Lin P and Zhang L: Localization of peripheral pulmonary lesions to aid surgical resection: A novel approach for electromagnetic navigation bronchoscopic dye marking. Eur J Cardiothorac Surg 52: 516-521, 2017.

7. Han Y, Kim HJ, Kong KA, Kim SJ, Lee SH, Ryu YJ, Lee JH, Kim Y, Shim SS and Chang JH: Diagnosis of small pulmonary lesions by transbronchial lung biopsy with radial endobronchial ultrasound and virtual bronchoscopic navigation versus CT-guided transthoracic needle biopsy: A systematic review and meta-analysis. PLoS One 13: e0191590, 2018.

8. Durakovic A, Andersen H, Christiansen A and Hammen I: Retrospective analysis of radial EBUS outcome for the diagnosis of peripheral pulmonary lesion: Sensitivity and complications. Eur Clin Respir J 2: 28947, 2015.

9. Kurimoto N, Miyazawa T, Okimasa S, Maeda A, Oiwa H, Miyazu Y and Murayama M: Endobronchial ultrasonography using a guide sheath increases the ability to diagnose peripheral pulmonary lesions endoscopically. Chest 126: 959-965, 2004.

10. Liu J, Han J, Lv H and Li B: An ultrasonic sensor system based on a two-dimensional state method for highway vehicle violation detection applications. Sensors (Basel) 15: 9000-9021, 2015.

11. Park J, Je Y, Lee H and Moon W: Design of an ultrasonic sensor for measuring distance and detecting obstacles. Ultrasonics 50: 340-346, 2010.

12. Paradis TJ, Dixon J and Tieu BH: The role of bronchoscopy in the diagnosis of airway disease. J Thorac Dis 8: 3826-3837, 2016.

13. Durheim MT, Kim S, Gulack BC, Burfeind WR, Gaissert HA, Kosinski AS and Hartwig MG: Mortality and respiratory failure after thoracoscopic lung biopsy for interstitial lung disease. Ann Thorac Surg 104: 465-470, 2017. 
14. Lieberman S, Gleason JB, Ilyas MIM, Martinez F, Mehta JP and Savage EB: Assessing the safety and clinical impact of thoracoscopic lung biopsy in patients with interstitial lung disease. J Clin Diagn Res 11: OC57-OC59, 2017.

15. Jo Y, Han DH, Beck KS, Park JS and Kim TJ: Practice pattern of transthoracic needle biopsy: 2016 survey in the members of Korean society of thoracic radiology. Korean J Radiol 18: 1005-1011, 2017

16. Anzidei M, Porfiri A, Andrani F, Di Martino M, Saba L, Catalano $\mathrm{C}$ and Bezzi $\mathrm{M}$ : Imaging-guided chest biopsies: Techniques and clinical results. Insights Imaging 8: 419-428, 2017.

17. Heerink WJ, de Bock GH, de Jonge GJ, Groen HJ, Vliegenthart R and Oudkerk M: Complication rates of CT-guided transthoracic lung biopsy: Meta-analysis. Eur Radiol 27: 138-148, 2017.

18. Accordino MK, Wright JD, Buono D, Neugut AI and Hershman DL: Trends in use and safety of image-guided transthoracic needle biopsies in patients with cancer. J Oncol Pract 11: e351-e359, 2015

19. Kim EJ and Kim KC: Utility of radial probe endobronchial ultrasound-guided transbronchial lung biopsy in diffuse lung lesions. Tuberc Respir Dis (Seoul) 82: 201-210, 2019.

20. Zhan P, Zhu QQ, Miu YY, Liu YF, Wang XX, Zhou ZJ, Jin JJ and $\mathrm{Li} \mathrm{Q}$ : Comparison between endobronchial ultrasound-guided transbronchial biopsy and CT-guided transthoracic lung biopsy for the diagnosis of peripheral lung cancer: A systematic review and meta-analysis. Transl Lung Cancer Res 6: 23-34, 2017.

21. Dhooria S, Sehgal IS, Gupta N, Aggarwal AN, Behera D and Agarwal R: Role of radial endobronchial ultrasound-guided transbronchial needle aspiration in the diagnosis of pulmonary nodules: Case report and literature review. Lung India 34: 61-64, 2017.

22. Steinfort DP, Khor YH, Manser RL and Irving LB: Radial probe endobronchial ultrasound for the diagnosis of peripheral lung cancer: Systematic review and meta-analysis. Eur Respir J 37: 902-910, 2011.
23. Hayama M, Izumo T, Chavez C, Matsumoto Y, Tsuchida T and Sasada S: Additional transbronchial needle aspiration through a guide sheath for peripheral pulmonary lesions that cannot be detected by radial EBUS. Clin Respir J 11: 757-764, 2017.

24. Boonsarngsuk V, Kanoksil W and Laungdamerongchai S: Diagnosis of peripheral pulmonary lesions with radial probe endobronchial ultrasound-guided bronchoscopy. Arch Bronconeumol 50: 379-383, 2014 (In English, Spanish).

25. Zhang Q, Zhang S, Xu X, Xu Q and Zhou J: Value of radial probe endobronchial ultrasound-guided transbronchial biopsy and computer tomography-guided transthoracic needle aspiration in the diagnosis of peripheral pulmonary lesions. Medicine (Baltimore) 96: e7843, 2017.

26. Zhang S, Zhou J, Zhang Q, Xu Q and Xu X: Diagnosis of peripheral lung diseases by combined measurement with ultrasound guidance under bronchoscope. Chin J Tuberc Respir 38: 566-569, 2015 (In Chinese).

27. Fukusumi M, Ichinose Y, Arimoto Y, Takeoka S, Homma C, Matsuoka H, Mouri A, Hamamoto Y, Matsumoto J and Kamimura M: Bronchoscopy for pulmonary peripheral lesions with virtual fluoroscopic preprocedural planning combined with EBUS-GS: A pilot study. J Bronchology Interv Pulmonol 23: 92-97, 2016

28. Hsia DW, Jensen KW, Curran-Everett D and Musani AI: Diagnosis of lung nodules with peripheral/radial endobronchial ultrasound-guided transbronchial biopsy. J Bronchology Interv Pulmonol 19: 5-11, 2012.

29. Eberhardt R, Anantham D, Ernst A, Feller-Kopman D and Herth F: Multimodality bronchoscopic diagnosis of peripheral lung lesions: A randomized controlled trial. Am J Respir Crit Care Med 176: 36-41, 2007.

This work is licensed under a Creative Commons Attribution-NonCommercial-NoDerivatives 4.0 International (CC BY-NC-ND 4.0) License. 